\title{
Batch and semibatch catalytic reactors (from theory to practice)
}

\author{
Gianni Donati ${ }^{\mathrm{a}, *}$, Renato Paludetto ${ }^{\mathrm{b}}$ \\ ${ }^{a}$ Snamprogetti S.p.A., Research and Development, Reactor and Modelling Department, Via Maritano, 26, 20097, S. Donato M.se, Milan, Italy \\ ${ }^{\mathrm{b}}$ EniChem S.p.A., Technology Planning, Research \& Technology, P.za Boldrini, 1, 20097, S. Donato M.se, Milan, Italy
}

\begin{abstract}
A short review of batch reactor theory and practice is the subject of this paper. Based on unsteady state mass and energy balances, the mathematical set of equations describing the time-dependent behaviour of a batch or semibatch reactor is reported. The attention is focused on some examples of batch reactor experiences (experimental studies, scale-up techniques, industrial operation and optimisation), in order to show how a theory-based sound engineering practice can lead to process improvements. (C) 1999 Elsevier Science B.V. All rights reserved.
\end{abstract}

Keywords: Batch reactor; Mathematical modelling; Mixing; Batch polymerisation; Gas-liquid interface; Emulsion polymerisation; Oxygen transfer; Fermentation; Oxides precipitation

\section{Introduction}

In the design of a reactor for chemical production, the first question, which has to be raised, is whether the process should be operated batchwise or continuously. In the large chemical industry the tendency is toward continuous operation; however, for many processes continuous operation would be unprofitable or even impossible. There is no general rule for the selection of the operation mode but economic balance, scale of production, long reaction times, flexibility of production, and nature of the process and the product may dictate the selection of batch or semibatch operation.

Economic balance. Fixed costs are generally lower for batch processes but they reach a break even at low production rates while continuous operation enjoys economies of scale for large productions. Variable

\footnotetext{
*Corresponding author. Tel.: +39-2-9307192; fax: +39-2-799644 E-mail address: donati.igd@uli.it (G. Donati)
}

costs are generally higher for batch processes because of starting and stopping operation, slack times, heat recovery difficulties, cost of personnel and control of operation.

Production scale. Batch reactors are therefore often used for small production rates such as fine chemicals and specialities where reaction condition can be adjusted to product specification or quality.

Long reaction times. This factor is more typical of biological fermentation that lasts some days compared to the relatively fast chemical reactions. In the fermentation case we can add the control of contamination, the delicate microorganism growth procedure and the possibility of manufacturing different products in the same reactors following market needs.

Production flexibility. The same reactor is often used not only for different products but also for different process operations such as heating, reacting, solvent evaporation, cooling, blending with additives, besides standard cyclic operations like reactor initial 
conditioning, gas evacuation, reactants charge, product discharge, reactor washing, etc.
The principle of conservation of matter can be written for each component $j$ in the following manner:

$\left\{\begin{array}{c}\text { accumulation } \\ \text { of mass of } j \text { in } \\ \text { the system }\end{array}\right\}=\left\{\begin{array}{c}\text { rate of mass } \\ \text { of } j \text { entering } \\ \text { the system }\end{array}\right\}-\left\{\begin{array}{c}\text { rate of mass } \\ \text { of } j \text { exiting } \\ \text { the system }\end{array}\right\}+\left\{\begin{array}{c}\text { rate of mass } \\ \text { of } j \text { produced } \\ \text { by reaction }\end{array}\right\}$.

Nature of process/product. The flexibility of batch reactors allows to adjust the reaction condition in various reaction phases and therefore to tailor the
The application of the principle of conservation of energy leads to an energy balance, which in general states that

$\left\{\begin{array}{c}\text { accumulation } \\ \text { of energy in } \\ \text { the system }\end{array}\right\}=\left\{\begin{array}{c}\text { rate of energy } \\ \text { entering the } \\ \text { system }\end{array}\right\}-\left\{\begin{array}{c}\text { rate of energy } \\ \text { exiting the } \\ \text { system }\end{array}\right\}+\left\{\begin{array}{c}\text { rate of energy } \\ \text { produced in } \\ \text { the system }\end{array}\right\}$.

process variables to process specifications. This is an additional reason to prefer batch operation, in some cases even for large-scale productions, as in the plastic industry.

Additional reasons, in favour of batch and semibatch operations, belong more to the $\mathrm{R} \& \mathrm{D}$ practice and attitude. Many reactions are first investigated in batch lab equipment and scaling up by enlarging vessels, without kinetic experiments or other engineering evaluations may appear as the easiest way. This theorem is however far to be proven especially when transport phenomena and mixing effects are relevant to the examined process.

In the following section, the theory of ideal batch reactors is recalled as a basis for simple macroscopic balance calculations. The application of microscopic balances is reported in order to show the related computational difficulties. As always is the case, the solution stays in the middle: a series of industrial cases is described in order to show how a theory-based sound engineering practice can lead to process improvements.

\section{Theory [1]}

We will try to present in a simple way the basis of mass and energy balances that are reported on chemical reaction engineering books and are useful for the computation of concentration, conversion and temperature profiles in a batch or semibatch reactor.
These balances can in principle be represented in different ways: composition can be referred in terms of mass, molar fraction, concentration or conversion while energy could be computed using different reference states (pure components or elements at a given temperature and pressure) and be specific to unit mass or unit mole. This problem may appear trivial but may lead to some misunderstanding. In fact moles follow reaction stoichiometry but total moles are not necessarily conserved. On the other hand mass density is usually assumed to be constant while total concentration can be variable with composition. Finally energy is easily referred to mass with the exemption of energy variations due to reaction. This is why some authors prefer to use mass and mass fractions instead of moles. We will hold on both for the sake of clarity and make use of simplifying hypotheses for the most common reaction cases. In Section 3 some applications of mass and energy balances will be shown together with additional example where these balances show their uselessness for the solution of important specific process problems. This is to underline the fact that an automatic system does not exist for process improvement and innovation.

\subsection{The well-mixed batch reactor}

This is the simplest case one can afford: no material is supplied to or withdrawn from the reactor during the reaction. The material balance of component $j$ 
becomes

$\frac{\mathrm{d} n_{j}}{\mathrm{~d} t}=R_{j} V$

where $n_{j}$ are the moles of component $j, V$ is the volume of the reactor mixture and $R_{j}$ is the molar rate of production per unit volume of component $j$

$R_{j}=\sum_{k=1}^{N R} v_{j k} r_{k}$,

where $v_{j k}$ is the stoichiometric coefficient of component $j$ in the $k$ reaction, $r_{k}$ is the molar rate of $k$ reaction and $N R$ the number of reactions. If the density of the mixture remains constant the balance Eq. (1) can be simplified as follows in terms of concentration of component $j, C_{j}$ :

$\frac{\mathrm{d} C_{j}}{\mathrm{~d} t}=R_{j}$.

In order to compute the reactor performance starting from given initial concentrations of reactants, Eq. (1) or Eq. (3) can be integrated analytically or numerically following the complexity of the reaction stoichiometry and kinetics involved in the process.

In practice mixing effects and temperature deviation influence the performance of a batch reactor. In addition in many cases the reactor is not operated batchwise and a feed of reactants and withdrawal of products is operated during the reaction.

\subsection{The semicontinuous isothermal batch reactor}

The method of gradual supply of reactants to a batch reactor is often adopted in practice. It is done for example in the case of reactions having a very high heat effect so as to keep the temperature within certain limits. In many biological fermentation reactions, nutritious matter is added at a predetermined rate in order to achieve optimum production. In some cases of equilibrium reactions, reaction products are removed with the purpose of increasing the degree of conversion. The material balance of the semicontinuos isothermal reactor for all components becomes

$\frac{\mathrm{d} n_{j}}{\mathrm{~d} t}=F^{\text {in }} C_{j}^{\text {in }}-F^{\text {out }} C_{j}^{\text {out }}+V R_{j}$,

where $F^{\text {in }}$ and $F^{\text {out }}$ are the volume flows, which can be variable during operation, at the inlet and outlet of the reactor and $R_{j}$ is a function of concentrations $C_{j}$ in the reactor that, the given well mixed hypothesis, are equal to $C_{j}^{\text {out }}$. Since $F^{\text {in }}$ and $F^{\text {out }}$ are still unspecified functions of time, a general solution to Eq. (4) cannot be given. In addition, the variation of the reactor volume $V$ has to be computed from a general mass balance that, in the case of constant density, is transformed into a volume balance

$\frac{\mathrm{d} V}{\mathrm{~d} t}=F^{\mathrm{in}}-F^{\text {out }}$.

In the case $F^{\text {in }}$ equals $F^{\text {out }}$ and, consequently, $V$ is constant, Eq. (4) can be written in terms of concentrations

$\frac{\mathrm{d} C_{j}}{\mathrm{~d} t}=\frac{F}{V}\left(C_{j}^{\mathrm{in}}-C_{j}\right)+R_{j}$.

In the case $F^{\text {out }}=0$ and $F^{\text {in }}=F=$ constant, Eqs. (5) and (4) become, respectively,

$V=V_{0}+F t$,

$\frac{\mathrm{d} n_{j}}{\mathrm{~d} t}=F^{\mathrm{in}} C_{j}^{\mathrm{in}}+\left(V_{0}+F t\right) R_{j}$.

In any case, the assumptions made are to be held in mind in order to guarantee mass conservation whatever variables are used.

\subsection{The non-isothermal reactor}

The application of the principle of energy conservation parallels that of mass conservation. Limiting us to thermal energy and work, this principle can be described by the following equation:

$\frac{\mathrm{d} U}{\mathrm{~d} t}=H^{\text {in }}-H^{\text {out }}+Q-W$,

where $U$ is the total internal energy, $H^{\text {in }}$ the enthalpy input, $H^{\text {out }}$ the enthalpy output, $Q$ the rate of heat supply and $W$ is the rate of work done. This general equation can be represented in different ways, following the reference state used for internal energy and enthalpy. For most practical calculation it is useful to assume as a reference state pure components at reference temperature $T_{0}$, to operate at constant pressure, and to neglect heats of mixing. Under these conditions Eq. (9) can be more conveniently written

$$
\begin{aligned}
\frac{\mathrm{d} \rho V C_{p} T}{\mathrm{~d} t}= & F^{\text {in }} \rho^{\text {in }} C_{p}^{\text {in }}\left(T^{\text {in }}-T_{0}\right)-F^{\text {out }} \rho^{\text {out }} C_{p}^{\text {out }}\left(T^{\text {out }}-T_{0}\right) \\
& +V \sum_{k} r_{k} \Delta H_{r k}+u A\left(T-T_{w}\right) .
\end{aligned}
$$


In the case of well-mixed batch reactor and $C_{p}$ independent of temperature and composition, the balance becomes

$$
\rho V C_{p} \frac{\mathrm{d} T}{\mathrm{~d} t}=+V \sum_{k} r_{k} \Delta H_{r k}+u A\left(T-T_{w}\right) .
$$

If the reactor is adiabatic and only one reaction occurs, referring to the key component $j$, we can combine energy and mass balance as follows:

$\frac{\mathrm{d} T}{\mathrm{~d} t}=+\frac{r \Delta H_{r}}{\rho C_{p}}=\frac{\Delta H_{r}}{v_{j} \rho C_{p}} \frac{\mathrm{d} C_{j}}{\mathrm{~d} t}$.

That is a linear dependence between temperature and concentration that can be found. In the case of the semibatch reactor with feed $F$ and no product withdrawal

$$
\begin{aligned}
\rho V C_{p} \frac{\mathrm{d} T}{\mathrm{~d} t}= & F \rho C_{p}\left(T^{\mathrm{in}}-T_{0}\right) \\
& +V \sum_{k} r_{k} \Delta H_{r k}+u A\left(T-T_{w}\right) .
\end{aligned}
$$

The energy balance equation has to be coupled with mass balances and the resulting system of differential equations solved simultaneously, given the initial conditions and the operating feed and heat exchange policy, in order to compute temperature and composition in the reactor.

\section{Practice}

Mass and energy balance equations are a basic tool for the design, scale up and simulation of batch reactors. The equations reported in the previous Section 2 are just examples of application of the principle of conservation of mass and thermal energy and more complex representations can be found following the specific reaction technology. When more than one phase is present as in gas-liquid, liquid-liquid or gas-liquid-solid systems, multiphase equilibria or interphase-intraphase transport phenomena may be taken into consideration.

However, in a large number of production processes, product and process requirements go far behind the simple mass and energy balance computation. The reasons why the reactor characterisation problem may become more intriguing and require additional investigation are many. First of all the reaction rate, as defined by Eq. (2), may not only be a state function of composition and temperature but may be strongly influenced by transport phenomena and degree of mixing. The whole performance of the reactor in terms of productivity, selectivity and product quality may be related to these factors that are in turn related to the reaction technology used. In the following we will try, using some examples taken from practice with real processes, to give a flavour of an engineering approach to an industrial problem and to show how the solution may be found outside the traditional rules.

Starting from some complex applications of mass and energy balance equations we will progressively enter a domain that is more strictly related to technology.

\subsection{Mixing with fast competitive reactions}

Let us start with an example showing how the concept of well-mixed tank reactor can be misleading with respect to the correct mathematical description of a batch reactor. The mathematical approach described in the previous section can be effectively used for the simulation of batch or semibatch reactors. In these cases, kinetics of reactions have a characteristic time some order of magnitude higher than the mixing time of the reactor itself, which is strictly related to the agitation degree achievable by the adopted stirring system. Let us now examine a case where mixing and reaction time can compete, giving rise to yield and selectivity values non-expected on the basis of the kinetics relationships characterising the reacting system. Let us suppose to have three fast, irreversible, isothermal second-order reactions
(i) $\mathrm{A}+\mathrm{B} \rightarrow \mathrm{T}$,
(ii) $\mathrm{A}+\mathrm{B} \rightarrow \mathrm{S}$,
(iii) $\mathrm{B}+\mathrm{C} \rightarrow \mathrm{Q}$,
$k_{1} \gg k_{2}>k_{3}$.

$\mathrm{A}$ and $\mathrm{C}$ are first loaded in the reactor with concentrations $C_{\mathrm{a}}^{0} \gg C_{\mathrm{c}}^{0}$, then $\mathrm{B}$ is added at a constant rate, with a total added amount nearly stoichiometric with respect to component A. The goal is to maximise the production of $\mathrm{T}$, trying to avoid the formation of $\mathrm{S}$ and $\mathrm{Q}$ using a suitable agitation system and possibly a more effective $\mathrm{B}$ addition policy.

The two reactions (i) and (ii) consuming A are parallel, so no effect of fluid dynamics can be expected 
on selectivity towards $\mathrm{T}$; in fact whatever B concentrations would be inside the reactor, for each mole of reacted $\mathrm{A}$, the moles of $\mathrm{T}$ and $\mathrm{S}$ will be always in the ratio $k_{1} / k_{2}$ and selectivity will be

$S e_{\mathrm{T}}=k_{1} /\left(k_{1}+k_{2}\right)$.

Some influence of fluid dynamics has to be expected on the reaction (iii) due to the presence of concentration gradients inside the reactors. In fact in the first time instant of $\mathrm{B}$ addition, the reaction rates and $\mathrm{T}$ selectivity will be

$$
\begin{aligned}
R_{1} & =k_{1} C_{\mathrm{a}}^{0} C_{\mathrm{b}}, \quad R_{2}=k_{2} C_{\mathrm{a}}^{0} C_{\mathrm{b}}, \quad R_{3}=k_{3} C_{\mathrm{c}}^{0} C_{\mathrm{b}}, \\
S e_{\mathrm{T}} & =k_{1} C_{\mathrm{a}}^{0} /\left(\left(k_{1}+k_{2}\right) C_{\mathrm{a}}^{0}+k_{3} C_{\mathrm{c}}^{0}\right) .
\end{aligned}
$$

So, even in the ideal case of perfectly mixed reactor, we must expect selectivity to drop with time because A is consumed more quickly than C; but also an effect of stirring speed may be expected because A consumption may lead to concentration gradients inside the reactors higher than those of C. In order to show the relative importance of the examined factors, three models have been developed and solved. The first one (Fig. 1(a)) is the well-mixed reactor, which could be taken as a reference point. The second one (Fig. 1(b)) is a two-stage reactor model, i.e. two reaction volumes, one simulating the reactor conditions close to the stirring device (impeller) and to the reactant injection point, the other one simulating the rest of the reactor. The two zones are connected by a volumetric liquid flow rate $Q_{\mathrm{r}}$ due to the agitation device. The third one (Fig. 1(c)) is a six-staged reactor, i.e. the reactor is simulated as a series of six reaction volumes, starting from the impeller and reactant addition one, connected together in a closed loop configuration by the recirculation flow induced by the stirring device.

For the sake of simplicity, we report the results of the different models for this set of parameter values: $k_{1} / k_{2} \cong 13 ; \quad k_{1} / k_{3} \cong 100 ; \quad C_{\mathrm{c}}^{0} / C_{\mathrm{a}}^{0} \cong 4$. The expected initial selectivity is $\mathrm{Se}_{\mathrm{T}} \cong 0.895$ for the well-mixed batch reactor. The mass balance Eq. (4) for each of the three reactants (which for products stoichiometric relationships can be applied) are solved over time with constant feed rate of component $\mathrm{B}$, and the results clearly show that the final value of selectivity $S e_{\mathrm{T}} \cong 0.857$ is achieved whatever feed rate, or time period of addition, is used. As for the well-mixed reactor model, also for the other two models (twostages and six-stages models) the relative time dependent mass balance equations are solved (three equations for each stage) by means of standard Runge-Kutta numerical procedure. The results are summarised in Fig. 2 where the mixing time is defined as the volume of the reactor divided by the internal recirculation flow rate $\left(\tau_{\mathrm{m}}=V / Q_{\mathrm{r}}\right)$. For the two-stage model, the performances of the batch reactor are calculated as a function of the time period of $\mathrm{B}$ addition, at five different mixing times that could be obtained by using different impellers and/or dif-

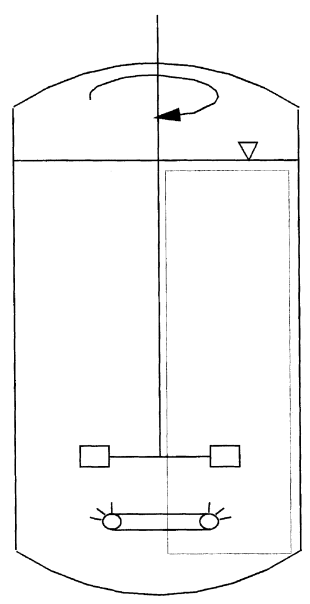

a) well mixed reactor

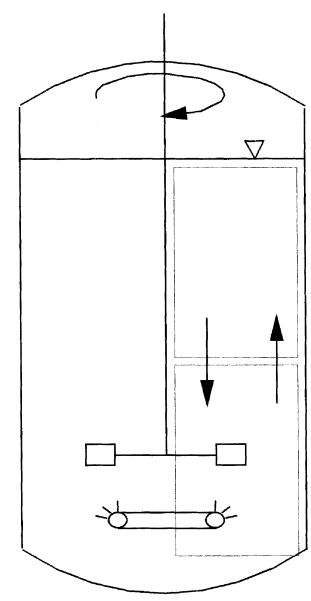

b) two stages reactor

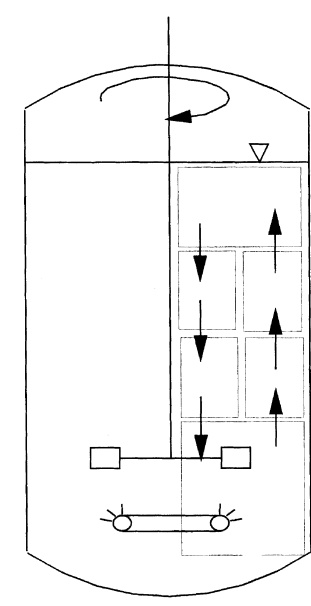

c) six stages reactor

Fig. 1. 


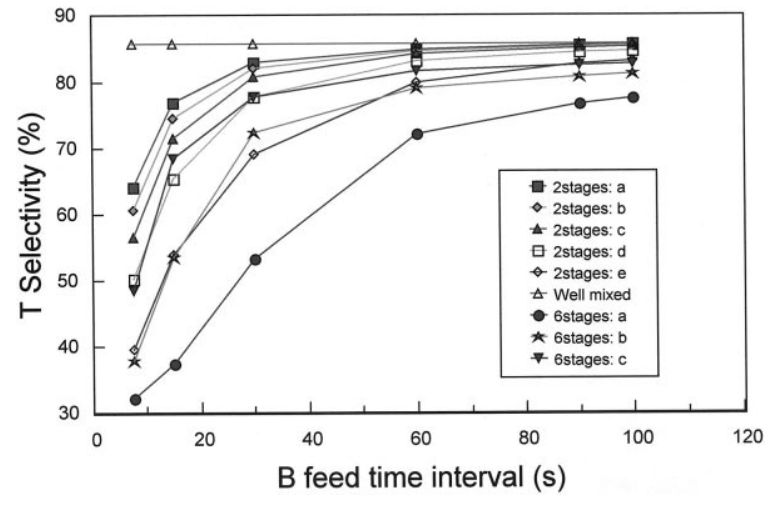

Fig. 2.

ferent rotational speed. In Fig. 2 the two-stage curves are obtained with equal reaction volumes and: curve a: $\tau_{\mathrm{m}}=21 ; \mathrm{b}: \tau_{\mathrm{m}}=25 ; \mathrm{c}: \tau_{\mathrm{m}}=30 ; \mathrm{d}: \tau_{\mathrm{m}}=33$ and e: $\tau_{\mathrm{m}}=67$. At low feed times, selectivity drops, while long $\mathrm{B}$ addition times favour selectivity to tend asymptotically to the value of the ideal well mixed case. This is true also when reducing the mixing time, i.e. with increasing internal recirculation, but reaching the asymptote at lower addition times. Thus we can conclude that in this case mixing favours selectivity and allows the reacting system to behave more closely to the ideal case. This is confirmed also with the six equal volume stages model, for which the cases $\mathrm{a}: \tau_{\mathrm{m}}=67$; $\mathrm{b}$ : $\tau_{\mathrm{m}}=33$ and $\mathrm{c}: \tau_{\mathrm{m}}=20$ are reported. As it can be easily seen, at equal mixing times the six-stage model exhibits a more pronounced non-ideal behaviour.

As a conclusion, key factors for the analysed reaction system are the mixing rate and the rate of reactant addition. Short feed times could in principle mean short batch time cycles and then higher productivity. This requires high recirculation rates, that could be obtained with different rotational regimes and/or different impeller design, provided that technical limits (cavitation, overflow from the reactor, etc.) are respected. This model can help to successfully choose the right combination of these two important factors.

\subsection{Batch polymerisation modelling}

The case of a semibatch radical exothermic copolymerisation has been analysed in order to build a mathematical model of the industrial scale reactor and the ancillary units, capable to predict the dynamic behaviour in terms of temperature, concentration, heat exchange and physico-chemical parameters of engineering interest for all the phases of the reactions recipe. The study deals with the co-polymerisation of styrene and acrylonitrile to give a SAN co-polymer. The reaction is performed in a stirred tank reactor in a semibatch mode, which is adding a cold mixture of comonomers and chain initiator. Temperature is kept constant during the addition of the monomers, and then it is raised to promote complete conversion of the less reactive monomer residuals. Heat exchange is crucial, so it requires an external loop with a recirculation pump and a heat exchanger (Fig. 3).

The reaction model is the standard radical polymerisation, which comprises the initiation reactions through thermal decomposition of a peroxide, the radical polymerisation of the two co-monomers through the propagation reaction, the termination reaction due to recombination of two active radicals. For the simulation of the well stirred tank reactor operating in the batch we have to solve the mass and heat balances Eqs. (4) and (9), taking into account that during the reaction, the feed of monomers and initiator is a function of time $F^{\mathrm{in}}(t)$ and $F^{\text {out }}$ is 0 . Standard integration algorithms can compute this system of differential equations. As reported in [2], the integration could be easily performed using spreadsheet programs like LOTUS 123 or MS EXCEL, together with the contemporary calculation of all the derived variables of interest (i.e. physico-chemical parameters, heat transfer coefficients, pressure drops, etc.). These programmes show to be powerful tools to obtain a solution to the problem, to make it easily modifiable and to provide a direct graphic representation.

The results of the application of the described approach are reported in Fig. 4 for a typical industrial recipe. In particular, the reported data show the result of a series of safety tests in which we make the hypothesis to run the reaction adiabatically (simulating a failure of the cooling system), feeding the system with monomers and initiator for $12 \mathrm{~min}$, more or less the time for the reactor to reach the maximum values of the monomer concentrations. The aim is to determine the maximum feed time interval that the system can support, avoiding too high temperatures without an external cooling system. 


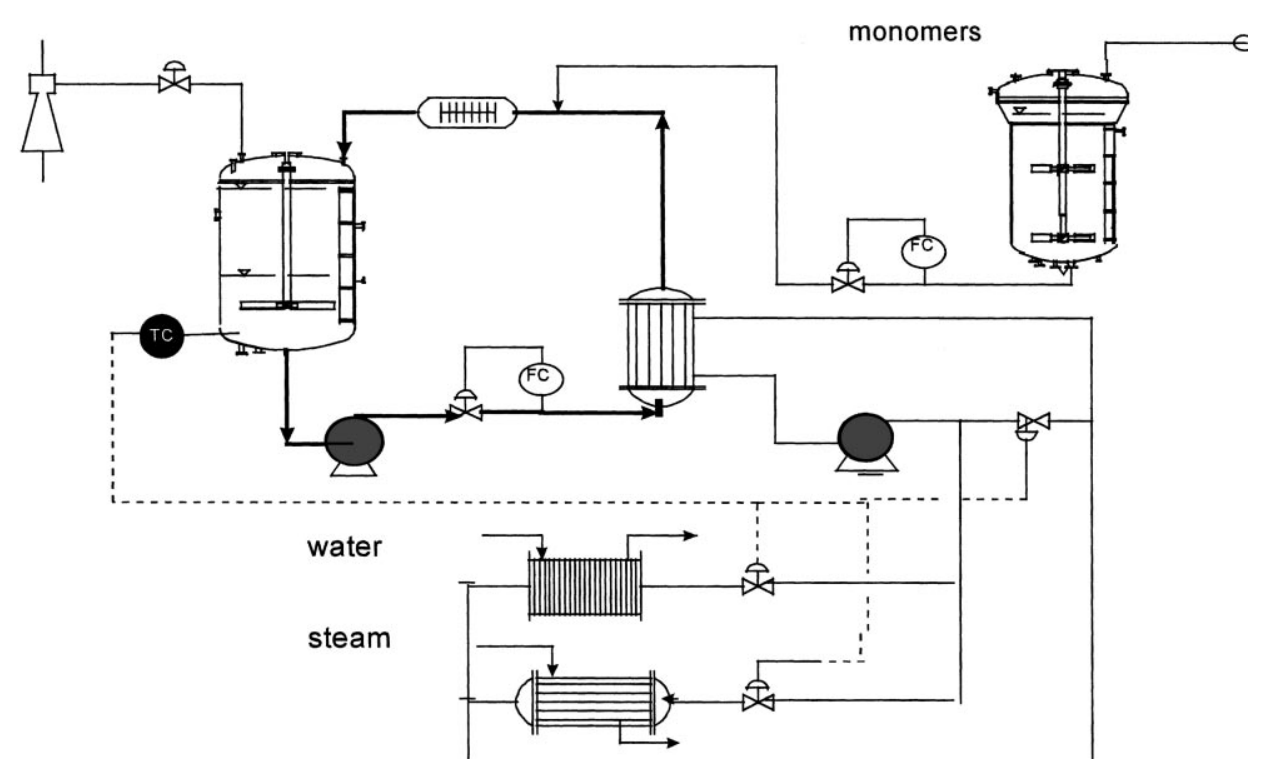

Fig. 3.

The first graph shows the simulated behaviour of co-monomer concentrations: the components first accumulate in the reactor due to the continuous constant feed, then they begin to react until, at the end of the addition, they are consumed by the polymerisation reaction. The second one shows the balance of the components in the reactor, as amount present in the reactor itself, and the total fed amount from the start of the addition. In the third graph the time evolution of initiator and radical concentrations is reported: initiator is fed together with co-monomers, tends to accumulate and decompose following the temperature path (fourth graph), giving rise to radicals that promote propagation reactions and gradually recombine through the termination reaction. Acrylonitrile is more reactive than styrene so its reaction could go to completion within the time interval of the simulation. Styrene on the contrary is still present in the system even at the end of the simulated time interval, due to the decay of radicals and initiators. Temperature goes through a minimum value that is due to the monomer temperature lower than that of the mass in the reactor, until the heat of reaction can first balance and then overcome the feed heat capacity. The analysed conditions give rise to a limit temperature of about $130^{\circ} \mathrm{C}$, which is in the limits allowed for the system.

\subsection{Gas-liquid emulsion polymerisation}

The emulsion polymerisation of tetrafluoroethylene is performed in semibatch reactors. The monomer is continuously fed in gaseous phase at high pressure while the polymer grows in the aqueous phase as latex. The polymerisation reaction is exothermic and temperature is controlled by cold water in the reactor jacket. At the start up of production a lot of problems came out regarding monomer purity, initiator addition policy, type and quantity of surfactants, temperature and pressure to be operated. These problems were investigated and a solution was found by a team of researchers and plant operators [3].

One key problem had no answer: the productivity and emulsion concentration obtained in the lab were not reproduced in 501 pilot; on the other hand the 5001 industrial autoclave showed a lower performance also in terms of product quality. The autoclaves had no internals and the stirrer had been selected on the basis of suppliers experience. In spite of the sophisticated mathematical models developed (initiation, radical propagation and polymer chain termination balances, molecular weight distribution, particle growth and population balances) the scale-up effect was not explained and productivity remained low and latex quality poor. The experimental evidence clearly 

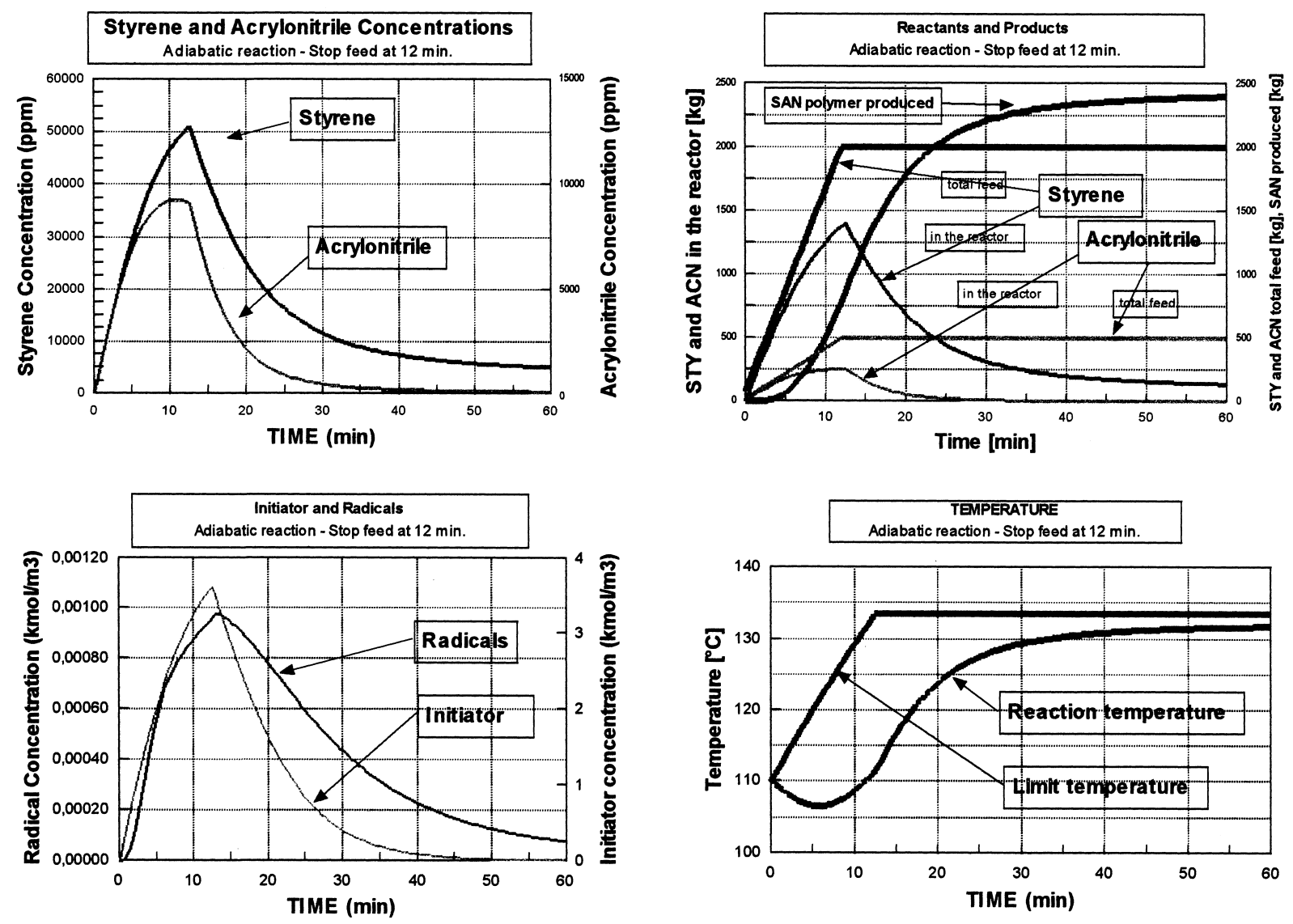

Fig. 4.

showed that increasing the stirrer speed allows increased productivity and at the same time decreased latex quality to final bulk coagulation and product destruction. The study has been therefore focused on reactor fluid dynamics and on the selection of stirring devices having low shear on the latex and capable of yielding a high gas-liquid area per unit volume.

Moke up reactors have been built for lab, pilot and industrial scale and the sulphite oxidation reaction was used for measuring the interphase area. The latex produced industrially was used for coagulation experiments. Fig. 5 shows schematically the interphase area obtainable in unbaffled reactors in a cavitating and non-cavitating situation.

Fig. 6 shows a comparison of interphase area obtainable with different stirrers and different speeds. Similar graphs together with coagulation experiments allowed the optimisation of stirrer shape that is a

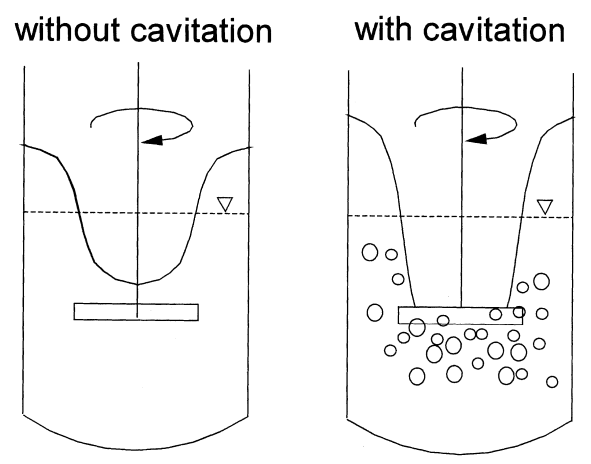

Fig. 5.

turbine with curved blades in order to reduce shear and vortexes on the blade in the face opposite to rotation. In addition, the height of the blade was preferred to diameter as it was recognised that large 


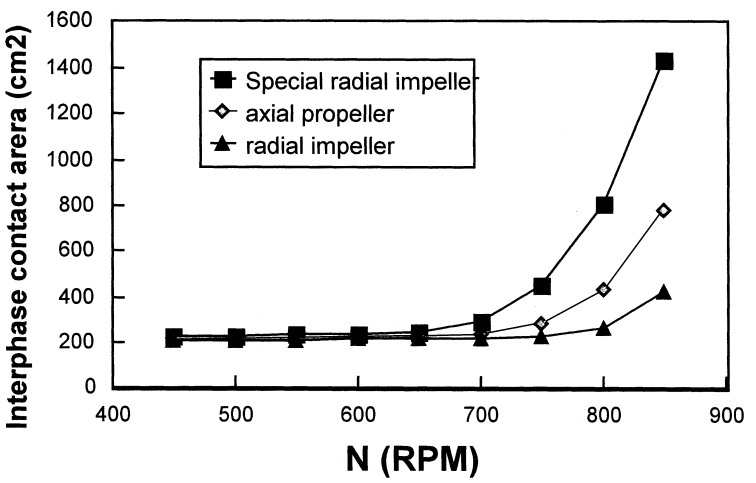

Fig. 6.

diameters were unfavourable for latex stability while the blade height could compensate for interfacial area. Experiments at the pilot scale produced the results of Fig. 7 where interphase area and, as a consequence, reactivity is reported for pilot and lab scale reactor.

The experimental results have been correlated using the Nagata theory [4] and the following scale-up rule has been obtained

$a=a_{\mathrm{c}}+K\left(1-\left(\frac{N}{N_{\mathrm{c}}}\right)^{2}\right)^{2} N^{3.2} d^{1.8}$,

where $a_{\mathrm{c}}$ and $N_{\mathrm{c}}$ are the critical area and speed when the vortex reaches the stirrer, $N$ the stirrer speed, $d$ the stirrer diameter, and $K$ is a constant experimentally determined for a given stirrer. A similar rule has been developed for latex stability in terms of time to reach coagulation.

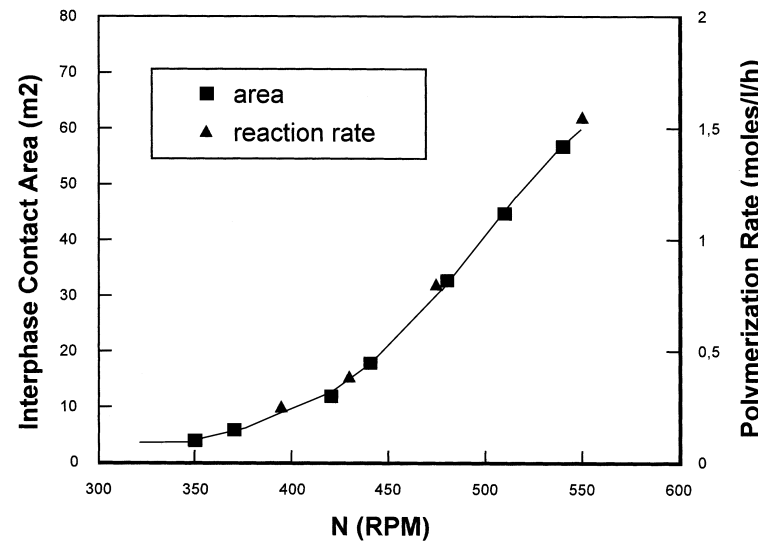

Fig. 7.
The investigation allowed the selection and design of the stirrer to be installed on the 5001 industrial reactor. The result has been an increase of 30\% productivity and latex concentration with a first class grade product.

The same concept has been used for the design of a new $3 \mathrm{~m}^{3}$ baffled reactor with completely different technology capable of an increase in $300 \%$ productivity with a product quality following market standards.

\subsection{Mixing and oxygen transfer in fermentation [5]}

Aerobic fermentation to produce antibiotics is a complex operation that is carried out in large reactors and last over one to two weeks (Fig. 8). The reactor is operated in semibatch mode and under strictly sterile conditions in order to avoid contamination and product loss. The main control parameters are aeration, agitation, temperature, $\mathrm{pH}$, dissolved oxygen $(D O)$ and respiration $\left(\mathrm{O}_{2}\right.$ and $\left.\mathrm{CO}_{2}\right)$, monitored through a series of chemical and physical analyses (sugar, ammonia, phosphate, viscosity, etc). After the broth and inoculum feed, these parameters control nutrients

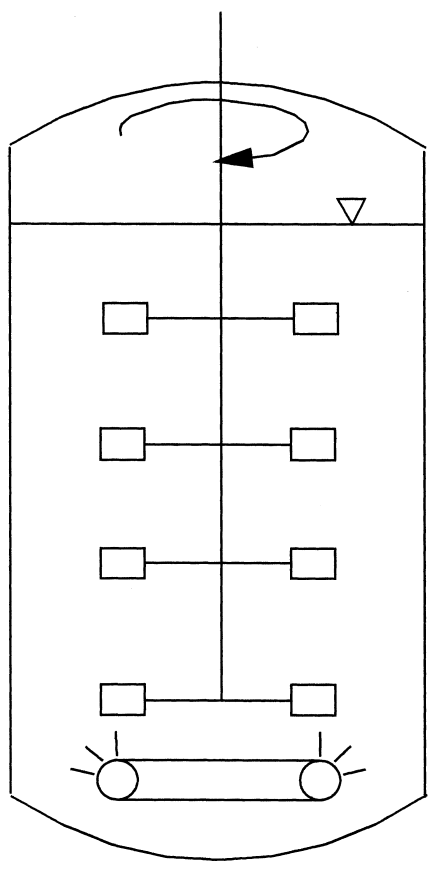

Fig. 8. 
feed and reactor operating conditions. The rules are well known by plant operators and have also been described in terms of mathematical models, that is mass and energy balances, where the key point is again the reaction rate expression. However, being the process evolution with time very slow, the study of performance of the system in the different stages may be more relevant. We have therefore focused the attention on mixing and oxygen transfer on large industrial fermentors that are the most important factors for a proper operation of real reactors and for the scale up from lab to industrial size equipment. In fact, due to the relevant height of large $100 \mathrm{~m}^{3}$ industrial reactors, pressure and oxygen profile may be quite different from top to bottom of the reactor. In static conditions and without fermentation the dissolved oxygen concentration $C^{*}$ varies linearly with the distance $Z$ from the liquid level

$C=\frac{1}{H e}\left(P_{0}+Z \rho_{L}(1-\Phi)\right) X$,

where $P_{0}$ is the total pressure in the ceiling, $X$ the molar fraction in the gas phase, $\rho_{\mathrm{L}}$ the liquid density and $\phi$ is the gas hold up. When the stirrer is activated, the action of the impellers gives rise to a certain liquid recirculation in the vessel and to the carry over of masses rich in oxygen from bottom to top and vice versa. The dissolved oxygen distribution is the result of two competing phenomena: mixing and mass transfer. Given the fermentor represented in Fig. 8, we can assume a fluid dynamic scheme of well-mixed reactors in series (Fig. 9) and write the oxygen mass balance in the liquid phase for each reactor

$$
\begin{aligned}
& Q\left(C_{2}-C_{1}\right)+K_{\mathrm{L}} a V\left(C_{1}^{*}-C_{1}\right)-N a V=0, \\
& Q\left(C_{3}+C_{1}-2 C_{2}\right)+K_{\mathrm{L}} a V\left(C_{2}^{*}-C_{2}\right)-N a V=0, \\
& Q\left(C_{4}+C_{2}-2 C_{3}\right)+K_{\mathrm{L}} a V\left(C_{3}^{*}-C_{3}\right)-N a V=0, \\
& Q\left(C_{3}-C_{4}\right)+K_{\mathrm{L}} a V\left(C_{4}^{*}-C_{4}\right)-N a V=0,
\end{aligned}
$$

where $Q$ and $q$ are the liquid and gas recirculation, $K_{\mathrm{L}} a$ is the mass transfer coefficient and $N a$ is the respiration rate supposed to be constant. Dividing by $Q$ we obtain the right-hand side part of the previous table, where $K$ is a dimensionless parameter which is the ratio of two characteristic times: the residence time $\tau=V / Q$ which represents the mixing capability of the stirrer and the gassing time $1 / K_{\mathrm{L}} a$ which represents

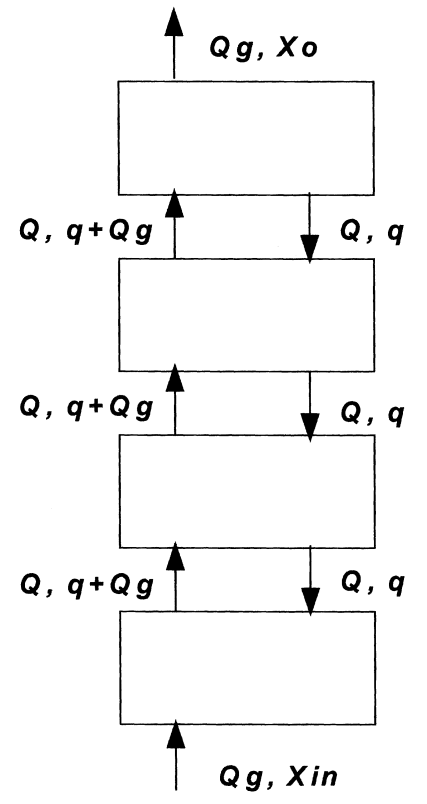

Fig. 9.

the mass transfer capability of the stirrer. $C^{*}$ is the equilibrium concentration. The solution of the linear system (14) is

$$
C_{i}=C_{i}^{\prime}-\frac{N a}{K_{L} a}, \quad i=1, \ldots, 4, \quad C_{i}^{\prime}=\sum_{j} A_{i, j} C_{j}^{*} .
$$

Expression (15) can be applied, provided that $N a$, the overall mass transfer coefficient $K_{\mathrm{L}} a$ and the liquid flow rate $Q$ are known. These values can

$$
\begin{aligned}
& \left(C_{2}-C_{1}\right)+K\left(C_{1}^{*}-C_{1}\right)-N a \tau=0, \\
& \left(C_{3}+C_{1}-2 C_{2}\right)+K\left(C_{2}^{*}-C_{2}\right)-N a \tau=0, \\
& \left(C_{4}+C_{2}-2 C_{3}\right)+K\left(C_{3}^{*}-C_{3}\right)-N a \tau=0, \\
& \left(C_{3}-C_{4}\right)+K\left(C_{4}^{*}-C_{4}\right)-N a \tau=0,
\end{aligned}
$$

be estimated from an overall balance of the gas phase and from literature correlation for the specific agitator used. This estimation has been made but here we prefer to focus the attention on experiments made on an industrial $112 \mathrm{~m}^{3}$ fermentor agitated by four Rushton impellers with a maximum power input per unit volume of $2.5 \mathrm{~kW} / \mathrm{m}^{3}$, as that represented in Fig. 8. 
A long probe has been inserted from a platform over the fermentor ceiling and temperature, velocity and dissolved oxygen profiles have been measured during a series of fermentation with two industrial strains of streptomyces aureofacies for the production of chlortetracycline and tetracycline. The axial $D O$ distribution is studied by plotting the mean oxygen concentration as a function of the distance from the bottom.

Fig. 10 shows $D O$ distribution measured within water and the computed values with different $K$ values: $K=\infty$ corresponds to negligible agitation effect compared to mass transfer, while, when $K=0$, the mass transfer is negligible compared to the mixing rate. In our fermentor $K$ is between 1 and 2 indicating that the two phenomena have the same order of magnitude. It is interesting to note that when $N=0$, that is when the fermentor is operated like an aerated tower, $K$ is less than 1 , indicating that mass transfer is greater than mixing time. In any case and in the absence of respiration the difference between $D O$ and its equilibrium value is zero in the centre of the fermentor.

Fig. 11 represents the $D O$ distribution for tetracycline at different ages while Fig. 12 shows the effect of agitation speed. It is interesting to note that the experimental lines are approximately parallel to the $C^{\prime}$ line, the difference being given by the driving force $N a / K_{\mathrm{L}} a$. The latest appears to be constant throughout the fermentor and changes with the growth of microorganisms and with the intensity of agitation. This fact

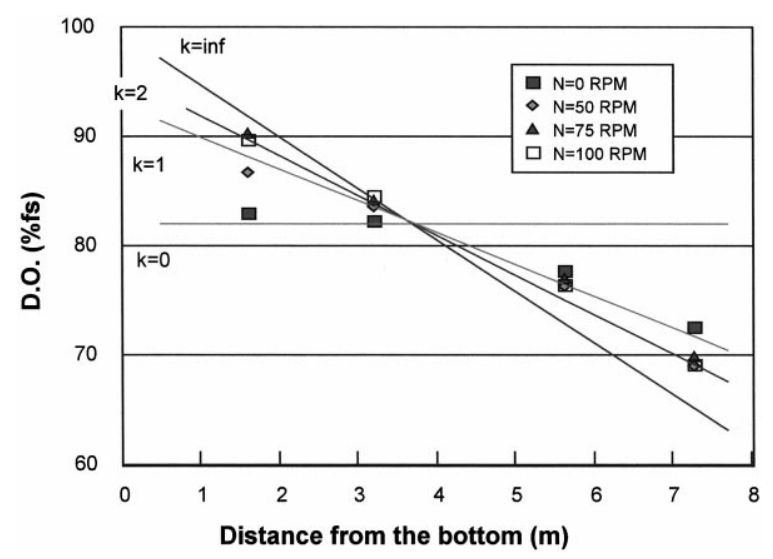

Fig. 10.

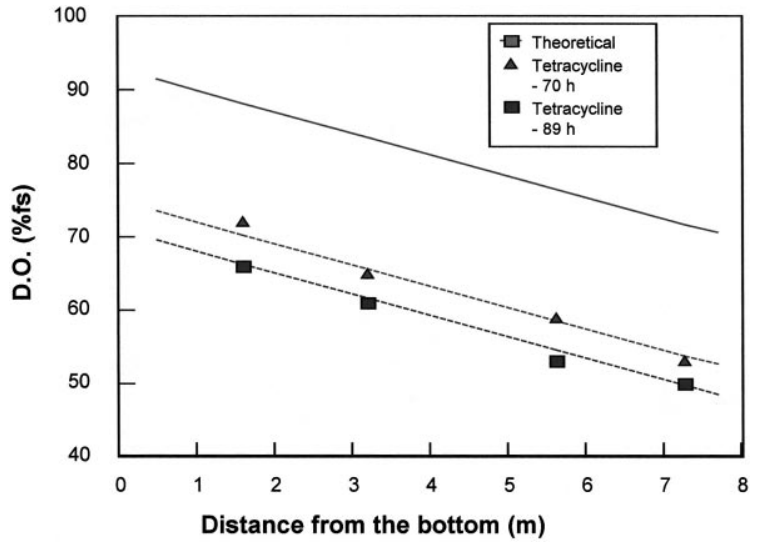

Fig. 11.

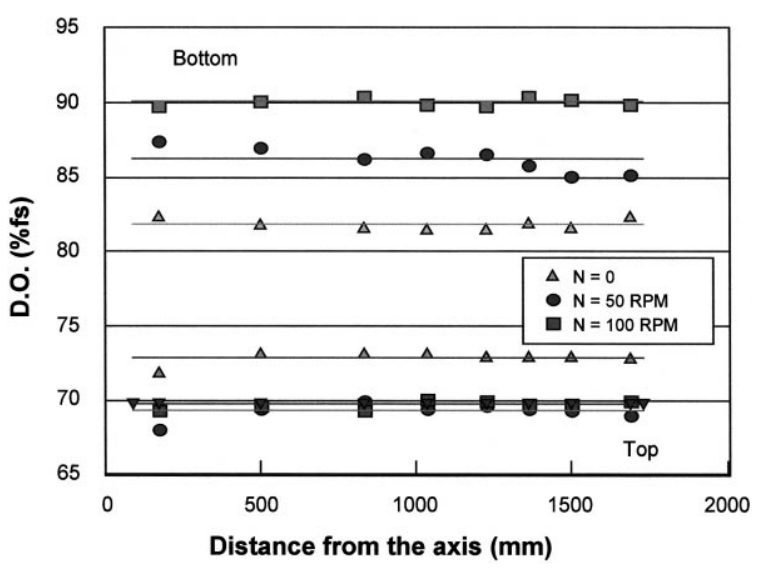

Fig. 12.

has many important implications for the process and for its operation:

- For a given fermentor the slope $K$ is a constant in water and during fermentation, that is only one measurement of $D O$ is necessary for the knowledge of oxygen distribution in the whole reactor.

- During fermentation this $D O$ profile translates, evidencing zones where $D O$ is lower than some critical value for respiration. It is possible to increase agitation, being $K$ constant, in order to increase $K_{\mathrm{L}} a$ and recover these undesired operating conditions.

- When scaling up a fermentation from lab to industrial size the fundamental difference in $D O$ between the two equipment, have to be kept in 
mind in order to design properly the industrial system without risking a bad performance and a loss of production.

In certain cases, where a constant and a specific value of $D O$ profile is needed, the selection of the most appropriate fermentation technology could be mandatory. We have seen how an aerated tower or an air lift reactor shows a $D O$ profile that is flat as compared to the turbine agitated vessel.

\subsection{Oxidative precipitation of inorganic pigments}

The production of oxyhydroxides and in particular of $\alpha-\mathrm{FeOOH}$ is an important process step for the synthesis of $\gamma-\mathrm{Fe}_{2} \mathrm{O}_{3}$ to be used for the fabrication of magnetic media. $\alpha-\mathrm{FeOOH}$ can be made by oxidative precipitation with an alkali and in the presence of air from ferrous sulphate aqueous solutions. The morphological characteristics of the $\gamma-\mathrm{Fe}_{2} \mathrm{O}_{3}$ precursor (crystal needle length, needle diameter/length) have a relevant effect on the magnetic properties of the product. Another important factor is repeatability of product properties from batch to batch. A typical preparation procedure is followed:

- The reactor is filled with $1 \mathrm{M}$ solution of ferrous sulphate.

- $\mathrm{NaOH}$ is added in order to precipitate $50 \%$ of $\mathrm{Fe}$ in the solution to $\mathrm{pH}=7.8$.

- Air flow is added to transform the brown hydroxide gel into the yellow pigment germ $\alpha-\mathrm{FeOOH}$ till a $\mathrm{pH}=3.5$ is reached.

- Under air the precipitation continues under controlled $\mathrm{pH}$ and temperature conditions.

- The precipitate is filtered, washed, dried and the powder is reduced in a furnace at $475^{\circ} \mathrm{C}$ and then oxidised at $300^{\circ} \mathrm{C}$ by a standard procedure.

The operation has been optimised on a lab scale traditional stirred reactor and it was soon evident that reaction times were long $(24 \mathrm{~h})$, repeatability was not guaranteed $( \pm 20 \%)$ and the quality was medium compared to market standards. The scale up on a $6 \mathrm{~m}^{3}$ reactor enlarged the times and showed problems with product quality. The oxidative precipitation lasted $42-45 \mathrm{~h}$, the intrinsic coercitivity of the powder was $\mathrm{Hci}=320-340 \mathrm{Oe}$ and the saturation magnetisation $\sigma_{\mathrm{s}}=70-74 \mathrm{emu} / \mathrm{g}$.

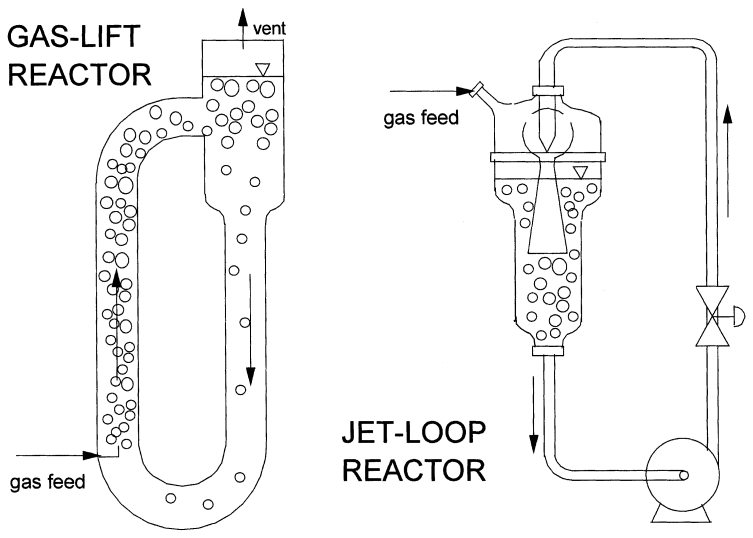

Fig. 13.

We soon realised that the viscosity of the fluid in the first phases of process was too high $(100000$ $200000 \mathrm{cP}$ ) for the traditional stirred reactor and that this technology was in any case poor for an efficient gas-liquid-solid reaction. We therefore made two pilot scale reactors based on jet loop and air lift technology [6] as shown in Fig. 13. The result was surprising and we succeeded in producing in $3 \mathrm{~h}$ the best available product in the market $(\mathrm{Hci}=420$ and $\sigma_{\mathrm{s}}=74 \mathrm{emu} / \mathrm{g}$ ). These values could vary following the recipe but repeatability was within less than $1 \%$.

\section{Conclusion}

A rationalisation in terms of mass and energy balance is presented for simple batch and semibatch reactors. Examples of application of the same principles to more complex situations have been shown and a possible use of spreadsheets is proposed as a mean of transferring mathematical models in an easy to handle form for plant operators. The examples clearly show how the job of scientists and engineers is not limited to descriptions in terms of mathematical models of reactor operation. The production of chemicals can be improved and even innovated by paying attention to phenomena that are relevant to the specific process and product and, case by case, by understanding, selecting or designing the best technology. This is the only way to keep scientist in the field working together and gain credibility and collaboration by industrial plant technicians. 


\section{References}

[1] G. Donati, R. Paludetto, Scale up of chemical reactors, Catal. Today 34 (1997) 483.

[2] R. Paludetto, Batch reactors modelling using spreadsheets, Proceedings of ECCE1 - Firenze (I), 1997.

[3] V. Arcella, G. Caputo, G. Donati, M. Gramondo, G. Santacasa, Ing. Chim. Ital. 18(3)(4) (1981).
[4] S. Nagata, Mixing Principles and Applications, Wiley, New York, 1975.

[5] R. Manfredini, V. Cavallera, Biotechnol. Bioeng. 25 (1893) 3115.

[6] M. Ghirardini, G. Donati, F. Rivetti, Chem. Eng. Sci. 47(9)(10)(11) (1992) 2209. 